\title{
Irregular Pyramids with Combinatorial Maps
}

\author{
Luc Brun ${ }^{1}$ and Walter Kropatsch ${ }^{2}$ \\ 1 LERI \\ I.U.T. Léonard de Vinci \\ Rue des Crayères \\ 51.350 Reims - France \\ Tel: (33) 3.26.91.30.88 \\ brun@leri.univ-reims.fr \\ WWw.univ-reims.fr/Labos/SciencesExa/Leri/membre/luc/ \\ 2 Institute for Computer-aided Automation \\ Pattern Recognition and Image Processing Group \\ Vienna Univ. of Technology \\ Favoritenstr. 9/1832 \\ A-1040 Vienna - Austria \\ krw@prip.tuwien.ac.at
}

\begin{abstract}
This paper presents a new formalism for irregular pyramids based on combinatorial maps. Such pyramid consists of a stack of successively reduced graph. Each smaller graph is deduced from the preceding one by a set of edges which have to be contracted or removed. In order to perform parallel contractions or removals, the set of edges to be contracted or removed has to verify some properties. Such a set of edges is called a Decimation Parameter. A combinatorial map encodes a planar graph thanks to two permutations encoding the edges and their orientation around the vertices. Combining the useful properties of both combinatorial maps and irregular pyramids offers a potential alternative for representing structures at multiple levels of abstraction.
\end{abstract}

\section{Introduction}

The multi-level representation of an image called pyramid 6 6 13] allows us to define a hierarchy in the different levels of representation of a same object. The method has been introduced by Pavlidis [6] who use a pyramid to define several partitions of a same image. Each connected component defined at one level is linked with its decomposition in the next level. His method defines a hierarchy between different partitions of the same image and is thus quite adapted to segmentation purpose where the definition of a region depends on the semantical context. For example, a face in an image may be considered as one region, or as the union of several regions defining the different semantical parts of the face such as the eyes and the hairs. The pyramids allow us to define a hierarchy between these two representations of a face which represent the same object at different levels. The first implementation of pyramids [6] use a regular tessellation of the image into a set of squares describing a balanced quadtree. Such a representation 
called a regular pyramid restricts the way in which objects defined at a given level are linked to their father in the next level. This restriction has been attenuated by Meer, Jolion and Montanvert [7] which have introduced a new family of pyramids called Irregular Pyramids. Kropatsch 9] has shown that irregular pyramids based on a pair of dual graphs may encode any partition of an image.

There are, at least, two ways to represent plane graphs: a pair of dual graphs $[12,8$ or combinatorial maps 1614 . In analogy to regular image pyramids, dual graph contraction [8] has been used to build irregular graph pyramids with the aim to preserve the high efficiency of the regular ancestors while gaining further flexibility to adapt their structure to the data. Experiences with connected component analysis [12, with universal segmentation [11], and with topological analysis of line drawings [10] show the great potential of this concept.

We have shown in [3] that an encoding of a planar map by a pair of dual graphs with an implicit encoding of the orientation may be converted into a combinatorial map encoding and conversely. Therefore, any object which may be described by 2D-combinatorial maps may also be described by dual graphs. However combinatorial maps present several advantages besides dual graphs which justify their use within the irregular pyramid framework:

- The combinatorial maps allow to encode a graph and its dual within a unique formalism. Moreover, the implementation also use a unique object to encode both graphs.

- The encoding of the orientation of the plane which is implicit within the dual graph formalism is explicit in the combinatorial map one.

- The combinatorial map formalism may be easily extended to higher dimensions 14. Thus using combinatorial maps pyramids, the same formalism may be used to define 2D, 3D or 4D Irregular Pyramids.

The rest of the paper is organized as follows: in Sect. 2 we give some definitions and basic properties of combinatorial maps together with the definition of the contraction and removal operations. In Sect. 3 we define the notion of decimation parameter which allows us to perform several contractions simultaneously. Finally, we give some perspectives opened by our work in Sect. 4 .

\section{Definition and Properties of Combinatorial Maps}

A combinatorial map [16] may be deduced from a planar graph by splitting each edge into two half edges called darts (see Fig. 1). The relation between two darts $d_{1}$ and $d_{2}$ associated to the same edge is encoded by the permutation $\alpha$ which maps $d_{1}$ to $d_{2}$ and vice-versa. The permutation $\alpha$ is thus an involution and its orbits are denoted by $\alpha^{*}(d)$, for a given dart $d$. These orbits encode the edges of the graph. Moreover, each dart is associated to a unique vertex. The sequence of darts encountered when turning around a vertex is encoded by the permutation $\sigma$. Using a counter-clockwise orientation, the orbit $\sigma^{*}(d)$ encodes the set of darts encountered when turning counter-clockwise around the vertex associated to the dart $d$. A combinatorial map can thus be formally defined by: 


\section{Definition 1. Combinatorial map}

A combinatorial map $G$ is the triplet $G=(\mathcal{D}, \sigma, \alpha)$, where $\mathcal{D}$ is a set called the set of darts and $\sigma, \alpha$ are two permutations defined on $\mathcal{D}$ such that $\alpha$ is an involution:

$$
\forall d \in \mathcal{D} \quad \alpha \circ \alpha(d)=d
$$

Given a dart $d, \alpha(d)$ and $\sigma(d)$ will be respectively called the $\alpha$ and $\sigma$-successors of $d$.

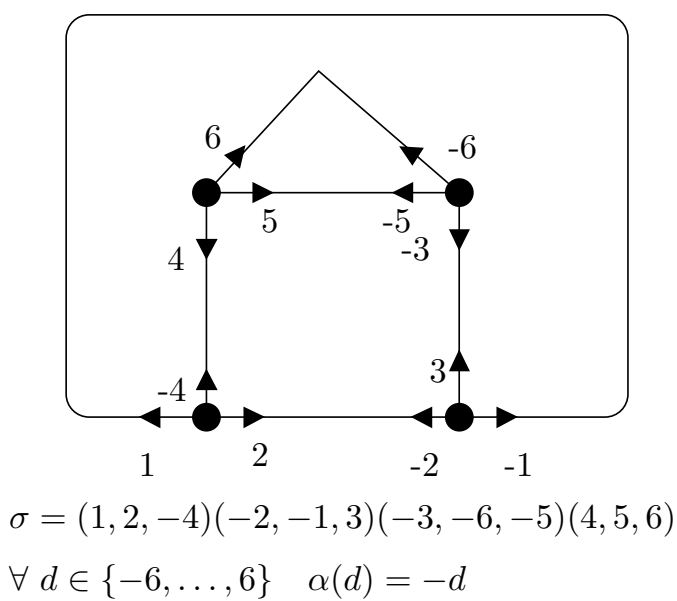

(a)

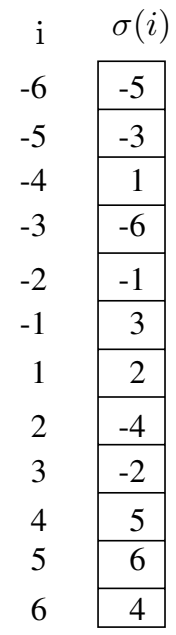

(b)

Fig. 1. Each dart of the combinatorial map is encoded by an integer. The permutation $\alpha$ associates its opposite to each dart. The permutation $\sigma$ (see (a)) is encoded by an array of integers (see (b)).

Note that, if the darts are encoded by positive and negative integers (see Fig. 1), the involution $\alpha$ may be implicitly encoded by the sign:

$$
\forall d \in \mathcal{D} \quad \alpha(d)=-d
$$

This convention is often used for practical implementation of connected combinatorial maps [1] where the combinatorial map is simply implemented by an array of integers encoding the permutation $\sigma$ (see Fig. 1(b)).

The permutations $\sigma$ and $\alpha$ allow us to pass from one dart to the other within a same connected component. Given a dart $d$, the set of darts of its connected component is denoted by $\mathcal{G} . d$ where $\mathcal{G}$ is the group of permutations generated by $\sigma$ and $\alpha$ : 


\section{Definition 2. Group associated to a combinatorial map}

Given a combinatorial map $G=(\mathcal{D}, \sigma, \alpha)$, the associated group $\mathcal{G}$ of $G$ is the subgroup generated by $\sigma$ and $\alpha$ within the symmetric group of all permutations on $\mathcal{D}$.

It is clear that two different labellings of the darts gives the same graph. This notion of proximity between combinatorial maps has been formalized by Gareth [5:

\section{Definition 3. Morphism between combinatorial maps}

Given two combinatorial maps $G_{1}=\left(\mathcal{D}_{1}, \sigma_{1}, \alpha_{1}\right), G_{2}=\left(\mathcal{D}_{2}, \sigma_{2}, \alpha_{2}\right)$ and their associated subgroups $\mathcal{G}_{1}$ and $\mathcal{G}_{2}$. A morphism $\phi: G_{1} \rightarrow G_{2}$ is a pair of functions $(\chi, \psi), \chi: \mathcal{G}_{1} \rightarrow \mathcal{G}_{2}$ and $\psi: \mathcal{D}_{1} \rightarrow \mathcal{D}_{2}$, where $\chi$ is a group homomorphism such that :

$$
\begin{aligned}
& \chi\left(\alpha_{1}\right)=\alpha_{2} \\
& \chi\left(\sigma_{1}\right)=\sigma_{2}
\end{aligned}
$$

and $\phi$ respects the orientation:

$$
\forall d \in \mathcal{D}_{1}\left\{\begin{array}{l}
\psi\left(\alpha_{1}(d)\right)=\alpha_{2}(\psi(d)) \\
\psi\left(\sigma_{1}(d)\right)=\sigma_{2}(\psi(d))
\end{array}\right.
$$

If $\psi$ is bijective $\phi$ will be called an isomorphism.

Note that, if $(\chi, \psi)$ is an isomorphism (1) may be rewritten as:

$$
\forall d \in \mathcal{D}_{1}\left\{\begin{array}{l}
\alpha_{1}(d)=\psi^{-1}\left(\alpha_{2}(\psi(d))\right) \\
\sigma_{1}(d)=\psi^{-1}\left(\sigma_{2}(\psi(d))\right)
\end{array}\right.
$$

For example if $\mathcal{D}_{1}$ and $\mathcal{D}_{2}$ are two sets of darts, and if $\pi$ is a bijective application from $\mathcal{D}_{1}$ to $\mathcal{D}_{2}$, we can show that the two combinatorial maps $G_{1}=$ $\left(\mathcal{D}_{1}, \sigma, \alpha\right)$ and $G_{2}=\left(\mathcal{D}_{2}, \pi \circ \sigma \circ \pi^{-1}, \pi \circ \alpha \circ \pi^{-1}\right)$, where $\sigma$ and $\alpha$ are defined on $\mathcal{D}_{1}$, are isomorphic.

Using the combinatorial map formalism, the dual of a combinatorial map $G=(\mathcal{D}, \sigma, \alpha)$ is deduced from $G$ by using $\varphi=\sigma \circ \alpha$ instead of $\sigma$ :

\section{Definition 4. Dual combinatorial map}

Given a combinatorial map $G=(\mathcal{D}, \sigma, \alpha)$, the combinatorial map $\bar{G}=$ $(\mathcal{D}, \varphi, \alpha)$ is called the dual of $G$. The permutation $\varphi$ is defined by:

$$
\varphi=\sigma \circ \alpha
$$

The orbits of $\varphi$ encode the faces of $G$.

The simplicity of the dual transformation avoids encoding explicitly the dual combinatorial map. Therefore, each operation performed on a combinatorial map will also modify its dual. This last point allows us to reduce the required memory and the complexity of our algorithms.

The removal operation (see Definition 5) is often used to simplify a graph. Using irregular pyramids, this operation may be performed to build the different levels of the pyramid from an initial graph. 


\section{Definition 5. Removal Operation}

Given a combinatorial map $G=(\mathcal{D}, \sigma, \alpha)$ and a dart $d \in \mathcal{D}$. If $\alpha^{*}(d)$ is not a bridge, the combinatorial map $G^{\prime}=\left(\mathcal{D}^{\prime}, \sigma^{\prime}, \alpha\right)=G \backslash \alpha^{*}(d)$ is defined by:

$-\mathcal{D}^{\prime}=\mathcal{D} \backslash \alpha^{*}(d)$ and

$-\sigma^{\prime}$ is deduced from $\sigma$ by:

$$
\forall d \in \mathcal{D}^{\prime} \quad \sigma^{\prime}(d)=\sigma^{n}(d) \text { with } n=\operatorname{Min}\left\{p \in \mathbb{N}^{*} / \sigma^{p}(d) \notin \alpha^{*}(d)\right\}
$$

Note that the bridges are excluded from Definition 5 in order to keep the number of connected components of combinatorial maps.

If combinatorial maps are used to encode a partition, the merge of two regions may be seen in two ways: first, it may be performed by removing one of the common boundary segments between the two regions. This operation is performed by the removal operation. In this case, each vertex of the combinatorial map is associated to the intersection of at least three boundaries (see Fig. 1). Secondly, the merge of the two regions may be performed by identifying the two regions and removing one of the edges encoding their adjacency. In this case, each vertex of the combinatorial map is associated to one region. This dual point of view on the merge of region is performed by the contraction operation (see Definition 6) which may be considered as the dual of the removal operation.

\section{Definition 6. Contraction operation}

Given a combinatorial map $G=(\mathcal{D}, \sigma, \alpha)$ and one dart d, in $\mathcal{D}$ which is not a self-loop. The contraction of dart $d$ creates the graph $G / \alpha^{*}(d)$ defined by:

$$
G / \alpha^{*}(d)=\overline{\bar{G} \backslash \alpha^{*}(d)}
$$

The expression of Definitions 5 and 6 in terms of modifications of the permutation $\sigma$ are given in $[3]$.

Note that the contraction operation is well defined since $d$ is a self-loop in $G$ iff it is a bridge in $\bar{G}$. Thus, any sequence of removal or contraction operations will preserve the number of connected components of the initial graph. This last property is useful in the irregular pyramid framework which attempts to simplify the initial planar map while preserving its essential structural properties such as the number of connected components.

\section{Decimation Parameters}

In order to perform more than one contraction simultaneously, we have to insure that the resulting combinatorial map is independent of the order of the contractions. We have shown 4 that if the contracted combinatorial maps remain connected, the contraction or the removal of any two darts is independent of the order of the operations

Thus if the contraction operations are allowed in a given order, they can be performed in any order. The contraction operations are well defined if we do not contract a self-loop. Using a Decimation Parameter (see Definition 8), the set of darts to be contracted form an Independent Vertex Set (see Fig. 22): 


\section{Definition 7. Independent Vertex Set}

Given a combinatorial map $G=(\mathcal{D}, \sigma, \alpha)$, a set of darts $\mathcal{D}^{\prime} \subset \mathcal{D}$ will be called an Independent Vertex Set iff:

$$
\alpha\left(\sigma^{*}\left(\mathcal{D}^{\prime}\right)\right) \cap \sigma^{*}\left(\mathcal{D}^{\prime}\right)=\emptyset
$$

The set $\mathcal{D}^{\prime}$ will be called a maximum Independent Vertex Set iff:

$$
\forall d \in \mathcal{D}-\sigma^{*}\left(\mathcal{D}^{\prime}\right) \quad \exists d^{\prime} \in \sigma^{*}(d) \quad \mid \quad \alpha\left(d^{\prime}\right) \in \sigma^{*}\left(\mathcal{D}^{\prime}\right)
$$

All vertices are defined by one dart of $\mathcal{D}^{\prime}$ or are linked to one vertex defined by a dart in $\mathcal{D}^{\prime}$.

Intuitively, the definition of an Independent Vertex Set consists to select a set of vertices called the set of surviving vertices and a set of edges such that the surviving vertices are not connected in the induced sub combinatorial map [3]. Then the selected edges connect a surviving vertex to a non surviving one. These edges become the edges to be contracted in the Decimation Parameter definition:

\section{Definition 8. Decimation Parameter}

Given a combinatorial map $G=(\mathcal{D}, \sigma, \alpha)$, a Decimation Parameter is a set of darts $\mathcal{D}^{\prime}$ such that $\mathcal{D}^{\prime}$ is an Independent Vertex Set of $G$ and:

$$
\forall d \in \mathcal{D}-\sigma^{*}\left(\mathcal{D}^{\prime}\right) \quad \exists ! d^{\prime} \in \mathcal{D}^{\prime} \quad \mid \quad \alpha\left(d^{\prime}\right) \in \sigma^{*}(d)
$$

The set $\mathcal{D}-\alpha^{*}\left(\mathcal{D}^{\prime}\right)$ is the set of surviving darts and is denoted $\mathcal{S D}$.

The definition of a Decimation Parameter, insures that the edges between surviving vertices are not contracted and that exactly one of multiple edges incident to a non surviving vertex is contracted. For example, the maximum Independent Vertex Set displayed in Fig. 22(a) does not satisfy the requirements of a Decimation Parameter since it contains a non-surviving vertex connected to two edges to be contracted. Fig. 2(b) sastisfies both requirements of an Independent Vertex Set and a Decimation Parameter. Thus, using the definition of a Decimation Parameter, no self-loops can be contracted and the contraction operations can be performed simultaneously.

Definition 9 defines a class of elementary paths called connecting paths which connect two surviving vertices. According to Definition 9, these paths contain exactly one dart which is not contracted. Therefore, the connecting paths may be denoted by $C P(d)$ where $d$ is the surviving dart of the path.

\section{Definition 9. Connecting Path}

Given a combinatorial map $G=(\mathcal{D}, \sigma, \alpha)$, a Decimation Parameter $\mathcal{D}^{\prime}$ of $G$ and two darts $b_{1}$ and $b_{2}$ in $\sigma^{*}\left(\mathcal{D}^{\prime}\right), C P\left(b_{1}, b_{2}\right)$ will be called a connecting path iff it is a path and if it verifies one of the following conditions:

1. The vertices $\sigma^{*}\left(b_{1}\right)$ and $\sigma^{*}\left(b_{2}\right)$ are adjacent:

$$
C P\left(b_{1}, b_{2}\right)=d \in \mathcal{S D}=\mathcal{D}-\alpha^{*}\left(\mathcal{D}^{\prime}\right)
$$

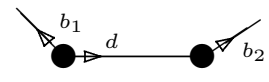

In this case the dart $d$ is a surviving dart. 


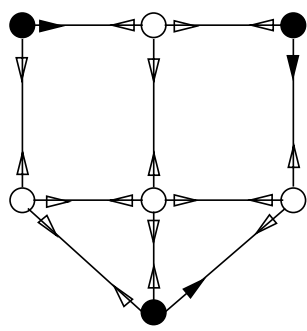

(a)

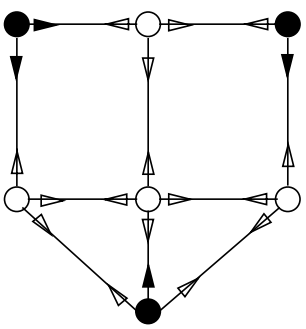

(b)

Fig. 2. A maximal Independent Vertex Set (a) and a Decimation Parameter (b). The set of contracted edges $\mathcal{D}^{\prime}$ is represented by black arrows. The surviving vertices are represented in black and have at least one dart in $\mathcal{D}^{\prime}$.

2. The vertices $\sigma^{*}\left(b_{1}\right)$ and $\sigma^{*}\left(b_{2}\right)$ are separated by one non surviving vertex:

$$
C P\left(b_{1}, b_{2}\right)=d_{1} d_{2} \quad \text { with: } \quad\left|\left\{d_{1}, d_{2}\right\} \cap \mathcal{S D}\right|=1
$$

In this case the non surviving vertex will be removed by the contraction of $d_{1}$ or $d_{2}$. Therefore, one of these dart must survive and the other be contracted.

3. The vertices $\sigma^{*}\left(b_{1}\right)$ and $\sigma^{*}\left(b_{2}\right)$ are separated by two non surviving vertices:

$$
C P\left(b_{1}, b_{2}\right)=d_{1} d_{2} d_{3} \quad \text { with }:\left|\left\{d_{1}, d_{2}, d_{3}\right\} \cap \mathcal{S D}\right|=1
$$

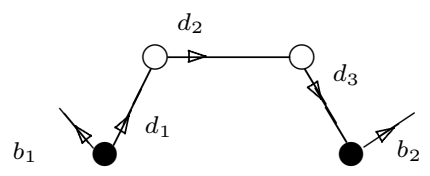

In this case the two non surviving vertices will be removed by the contraction of $d_{1}$ and $d_{3}$. The dart $d_{2}$ linking two non surviving vertices can not be contracted and is thus a surviving dart.

The set of surviving darts $\mathcal{S D}$ being symmetric by $\alpha, C P(d)$ and $C P(\alpha(d))$ are defined simultaneously for any dart $d$ in $\mathcal{S D}$. We can therefore, define the $\alpha$-successor of a connecting path $C P(d)$, denoted $\alpha_{C}(C P(d))$, as $C P(\alpha(d))$ :

\section{Definition 10. $\alpha$-Successor of Connecting Paths}

Given a combinatorial map $G=(\mathcal{D}, \sigma, \alpha)$, and a Decimation Parameter $\mathcal{D}$ ' of $G$, we define the involution $\alpha_{C}$ which associates to each connecting path containing one dart $d$ of $\mathcal{S D}$ the connecting path which contains $\alpha(d)$ :

$$
\forall d \in \mathcal{S D} \quad \alpha_{C}(C P(d))=C P(\alpha(d))
$$


We have shown in 4] that given a dart $d$ in $\mathcal{D}$, the sequence of darts $d, \varphi(d), \varphi^{2}(d)$ contains at least one non-contracted dart in $\mathcal{S D}$. The first dart of this sequence which is not contracted is called the representative dart $\operatorname{Rep}(d)$ of $d$ :

\section{Definition 11. Representative Dart}

Given a combinatorial map $G=(\mathcal{D}, \sigma, \alpha)$ and a Decimation Parameter $\mathcal{D}^{\prime}$, the representative dart $\operatorname{Rep}(d)$ of any dart $d$ in $\mathcal{D}$ is defined by:

$$
\operatorname{Rep}(d)=\varphi^{i}(d) \quad \text { with } \quad i=\operatorname{Min}\left\{j \in\{0,1,2\} \quad \mid \quad \varphi^{j}(d) \in \mathcal{S D}\right\}
$$

and is a non contracted dart belonging to $\mathcal{S D}$

Using the function $R e p$, we can define the $\sigma$-successor of each connecting path by:

Proposition 1. Given a combinatorial map $G=(\mathcal{D}, \sigma, \alpha)$ and a Decimation Parameter $\mathcal{D}^{\prime}$, the application:

$$
\sigma_{C}(C P(d))=C P(\operatorname{Rep}(\sigma(d)))
$$

defines a permutation on the set of connecting paths.

The proof of this proposition may be found in [4].

If we denote by $\mathcal{D}_{C}$ the set of connecting paths defined by a Decimation Parameter $\mathcal{D}^{\prime}$, the involution $\alpha_{C}$ and the permutation $\sigma_{C}$ define a combinatorial map on the set of connecting paths:

\section{Definition 12. Connecting Path Map}

Given a combinatorial map $G=(\mathcal{D}, \sigma, \alpha)$, and a Decimation Parameter $\mathcal{D}^{\prime}$, the set of connecting path $\mathcal{D}_{C}$, may be defined by:

$$
\mathcal{D}_{C}=\{C P(d), d \in \mathcal{S D}\}
$$

The map of connecting paths associated to the Decimation Parameter $G_{C}$ is defined by:

$$
G_{C}=\left(\mathcal{D}_{C}, \sigma_{C}, \alpha_{C}\right)
$$

(see Definition 10 and Proposition 11).

Since each connecting path is uniquely defined by one dart in $\mathcal{S D}$, we can consider $C P$ as a bijective application which associates to each dart in $\mathcal{S D}$ its associated connecting path. Then, if $G^{\prime}=\left(\mathcal{S D}, \sigma^{\prime}, \alpha^{\prime}\right)$ denotes the contracted combinatorial map $G / \alpha^{*}\left(\mathcal{D}^{\prime}\right)$, and if $\chi$ denote the application:

$$
\chi\left(\begin{array}{rl}
\mathcal{G}^{\prime} & \rightarrow \mathcal{G}_{C} \\
\alpha^{\prime} & \mapsto \alpha_{C} \\
\sigma^{\prime} & \mapsto \sigma_{C}
\end{array}\right.
$$

where $\mathcal{G}^{\prime}$ and $\mathcal{G}_{C}$ respectively denote the group of permutations associated to $G^{\prime}$ and $G_{C}$ (see Definition $[3$ ). 
The application $(\chi, C P)$ is an isomorphism between the contracted map $G^{\prime}$ and the connecting path map $\S G_{C}$ (see proof in [4]). Then, the permutations $\sigma^{\prime}$ and $\alpha^{\prime}$ may be respectively deduced form the permutation $\sigma_{C}$ and $\alpha_{C}$ as follow $($ see $(2)$ :

$\forall d \in \mathcal{D}-\alpha^{*}\left(\mathcal{D}^{\prime}\right)\left\{\begin{array}{l}\left.\alpha^{\prime}(d)=C P^{-1}\left(\alpha_{C}(C P(d))\right)=C P^{-1}(C P(\alpha(d)))\right)=\alpha(d) \\ \sigma^{\prime}(d)=C P^{-1}\left(\sigma_{C}(C P(d))\right)=C P^{-1}(C P(\operatorname{Rep}(\sigma(d))))=\operatorname{Rep}(\sigma(d))\end{array}\right.$

Therefore, the contracted combinatorial map $G^{\prime}=G / \alpha^{*}\left(\mathcal{D}^{\prime}\right)$ may be constructed from $G$, by leaving the permutation $\alpha$ unchanged and by computing for each surviving dart the value $\operatorname{Rep}(\sigma(d))$ i.e. by searching the minimal integer $j$ in $\{0,1,2\}$ such that $\varphi^{j}(\sigma(d))$ belongs to $\mathcal{S D}$. If this operation is performed in parallel on each surviving dart, the contracted combinatorial map may be built in constant time.

\section{Conclusion and Perspectives}

We have defined in this article the theorical framework needed to perform removal or contraction operations on combinatorial maps. The contraction operation is then generalized thanks to the definition of Decimation Parameter. These definitions allow us to design several contractions in parallel.

The definition of a contraction kernel by labeled pyramids is under development. This expected result together with the ones resumed in this article should allow us to study interesting applications of our model such as: segmentation [1, 2, efficient structural matching [15] or integration of moving objects. Finally, the extension of our model to higher dimensional spaces (3D) should be studied.

\section{References}

[1] J. P. Braquelaire and L. Brun. Image segmentation with topological maps and inter-pixel representation. Journal of Visual Communication and Image representation, 9(1), 1998.

[2] L. Brun, J.P. Domenger, and J.P. Braquelaire. Discrete maps : a framework for region segmentation algorithms. In Workshop on Graph based representations, Lyon, April 1997. published in Advances in Computing (Springer).

[3] L. Brun and Walter Kropatsch. Dual contraction of combinatorial maps. In GbR99. IAPR TC15, 1999.

[4] L. Brun and Walter Kropatsch. Dual contractions of combinatorial maps. Technical Report 54, Institute of Computer Aided Design, Vienna University of Technology, lstr. 3/1832,A-1040 Vienna AUSTRIA, January 1999. avalaible through http://www.prip.tuwien.ac.at/.

[5] A. Jones Gareth and David Singerman. Theory of maps on orientable surfaces. volume 3, pages 273-307. London Mathematical Society, 1978.

[6] S.L. Horowitz and T. Pavlidis. Picture segmentation by a tree traversal algorithm. Journal of The Association for Computing Machinery, 23(2):368-388, April 1976. 
[7] J.M. Jolion and A. Montanvert. The adaptative pyramid : A framework for $2 \mathrm{~d}$ image analysis. Computer Vision, Graphics, and Image Processing, 55(3):339-348, May 1992.

[8] Walter G. Kropatsch. Building Irregular Pyramids by Dual Graph Contraction. IEE-Proc. Vision, Image and Signal Processing, Vol. 142(No. 6):366-374, December 1995 .

[9] Walter G. Kropatsch. Equivalent Contraction Kernels and The Domain of Dual Irregular Pyramids. Technical Report PRIP-TR-42, Institute f. Automation 183/2, Dept. for Pattern Recognition and Image Processing, TU Wien, Austria, 1995.

[10] Walter G. Kropatsch. Property Preserving Hierarchical Graph Transformations. In Carlo Arcelli, Luigi P. Cordella, and Gabriella Sanniti di Baja, editors, Advances in Visual Form Analysis, pages 340-349. World Scientific Publishing Company, 1998.

[11] Walter G. Kropatsch and Souheil BenYacoub. Universal Segmentation with PIRRamids. In Axel Pinz, editor, Pattern Recognition 1996, Proc. of 20th ÖAGM Workshop, pages 171-182. OCG-Schriftenreihe, Österr. Arbeitsgemeinschaft für Mustererkennung, R. Oldenburg, 1996. Band 90.

[12] Walter G. Kropatsch and Herwig Macho. Finding the structure of connected components using dual irregular pyramids. In Cinquième Colloque DGCI, pages 147-158. LLAIC1, Université d'Auvergne, ISBN 2-87663-040-0, September 1995.

[13] M.D. Levine. Vision in man and machine. Mc Graw-Hill Book Compagny, 1985.

[14] Lienhardt. Subdivisions of n-dimensional spaces and n-dimensional generalized maps. In Annual ACM Symposium on Computational Geometry, all, volume 5, 1989.

[15] Jean-Gerard Pailloncy, Walter G. Kropatsch, and Jean-Michel Jolion. Object Matching on Irregular Pyramid. In Anil K. Jain, Svetha Venkatesh, and Brian C. Lovell, editors, 14th International Conference on Pattern Recognition, volume II, pages 1721-1723. IEEE Comp.Soc., 1998.

[16] W.T. Tutte. Graph Theory, volume 21. Addison-Wesley, encyclopedia of mathematics and its applications edition, 1984. 